\title{
Dictynna
}

Dictynna

Revue de poétique latine

10 | 2013

Varia

\section{Pari e superiore agli dèi: due esempi di retorica del potere nell'opera dell'esilio di Ovidio.}

\section{Luigi Galasso}

\section{(2) OpenEdition}

Journals

Édition électronique

URL : http://journals.openedition.org/dictynna/989

DOI : $10.4000 /$ dictynna.989

ISSN : 1765-3142

\section{Référence électronique}

Luigi Galasso, « Pari e superiore agli dèi: due esempi di retorica del potere nell'opera dell'esilio di Ovidio. », Dictynna [En ligne], 10 | 2013, mis en ligne le 18 décembre 2013, consulté le 10 septembre 2020. URL : http://journals.openedition.org/dictynna/989; DOI : https://doi.org/10.4000/dictynna.989

Ce document a été généré automatiquement le 10 septembre 2020.

\section{(c) (i) (9)}

Les contenus des la revue Dictynna sont mis à disposition selon les termes de la Licence Creative Commons Attribution - Pas d'Utilisation Commerciale - Pas de Modification 4.0 International. 


\title{
Pari e superiore agli dèi: due esempi di retorica del potere nell'opera dell'esilio di Ovidio.
}

\author{
Luigi Galasso
}

1 Nelle opere dell'esilio Ovidio costruisce, ai suoi fini, le componenti di un'ideologia del potere imperiale, che acquisiranno un carattere di modello fondante per i letterati che verranno dopo di lui. Da un lato è oggettivamente testimone di un mutato clima politico e culturale; dall'altro, la necessità in cui si trova lo spinge a lavorare sui modelli di comportamento che sono proposti dall'ideologia augustea'. In questo modo può costruire una difesa della sua posizione che sia ineccepibile dal punto di vista di chi gli ha inflitto la condanna. Per farlo ricorre a differenti strategie: una di queste consiste nell'imporre al sovrano, come vedremo, le conseguenze che derivano dall'applicazione delle affermazioni che compongono la sua immagine pubblica, e nello svilupparne gli esiti impliciti. Il poeta interviene in modo diversificato su elementi di vario ordine. Se si volesse provare ad individuare una sua visione del potere imperiale, si potrebbero tracciare significative linee di tendenza che definiscono un quadro coerente, ma nel contempo non ci si deve sorprendere se alcuni motivi, in apparente contrasto, vengano utilizzati a seconda delle necessità dell'argomentazione. Questo vale non solo per le tematiche legate al potere, ma anche, ad esempio, per quelle relative all'amicizia, e in questo senso si è parlato opportunamente di un uso 'retorico' della filosofia1. I singoli elementi hanno importanti precedenti o sono alle volte addirittura proverbiali, ma Ovidio imprime ad essi un conio e vi conferisce una memorabilità che ne determinerà il riutilizzo e lo sviluppo. Ne considereremo, mettendone in evidenza $\mathrm{i}$ precedenti, due apparentemente contraddittori, che sono uniti dal fatto che si riferiscono entrambi alla giustizia: (I) nell'esercizio della giustizia l'imperatore ha gli stessi limiti degli dèi ; (II) Augusto è invece più giusto degli dèi.

2 (I) Augusto, assorbito dalle numerose cure del suo impero, non ha avuto modo di leggere l'Ars, che è uno dei due capi di imputazione per la condanna all'esilio. Il concetto conosce una formulazione notevole in tr. 2, 213-218 : 
Fas ergo est aliqua caelestia pectora falli, et sunt notitia multa minora tua ;

utque deos caelumque simul sublime tuenti non vacat exiguis rebus adesse Iovi,

de te pendentem sic dum circumspicis orbem, effugiunt curas inferiora tuas.

3 È stato evidenziato il rapporto tra questi versi e l'inizio dell'Epistola ad Augusto di Orazio: tanti sono i gravi impegni dell'imperatore, che al poeta sembrerebbe di commettere una colpa nei confronti del bene comune se lo distraesse con i suoi scritti. Ovidio, che inizia la sua argomentazione con le parole citate, prosegue poi elencando tutte le attività e i problemi del sovrano. Da ciò risulta che è davvero impensabile che Augusto possa essere un lettore di poesia elegiaca e in particolare dell'Ars amatoria, che è stata causa della condanna alla relegatio ${ }^{2}$.

4 Significativamente, il motivo è riproposto in un'epistola delle Ex Ponto, questa volta però per indurre all'azione il destinatario, Messalino : parte della disgrazia dell'esule deriva dal fatto che Augusto ignora quale sia la situazione di Ovidio in mezzo ai barbari $(1,2,71-77)$ :

Nescit enim Caesar, quamvis deus omnia norit, ultimus hic qua sit condicione locus.

Magna tenent illud numen molimina rerum, haec est caelesti pectore cura minor,

nec vacat, in qua sint positi regione Tomitae, quaerere (finitimo vix loca nota Getae),

aut quid Sauromatae faciant ...

In questo caso i grandi impegni del sovrano impongono l'intervento di qualcuno che gli renda nota la condizione del poeta ${ }^{3}$. In seguito, all'interno di un'invocazione agli dèi, Augusto viene definito il più giusto di essi (97). Si recupera dunque nuovamente, anche se in maniera indiretta, la connessione con il tema della giustizia ${ }^{4}$. Può essere però interessante il modo in cui il concetto ricorre in un problematico passo ciceroniano.

Nell'orazione in difesa di Sesto Roscio di Ameria, Cicerone mette in rilievo come Silla, impegnato in occupazioni di assoluta rilevanza, quali la rifondazione e la risistemazione dello stato romano, non sia in grado di seguire ogni minimo dettaglio di ciò che accade. L'intento dell'oratore è naturalmente quello di distanziare il più possibile la posizione del dittatore da quella del suo liberto Crisogono. L'argomentazione è sviluppata verso l'inizio dell'orazione (22) ; viene poi ripresa con un paragone molto articolato tra i compiti di Giove e il suo conseguente modo di operare, e il parallelo comportamento di Silla.

[130] Quae omnia [le azioni compiute da Crisogono contro Sesto Roscio] si, quemadmodum solent liberti nequam et improbi facere, in patronum suum voluerit conferre, nihil egerit [scil. Chrysogonus]; nemo est enim qui nesciat propter magnitudinem rerum multa multos partim improbante, partim imprudente L. Sulla commisisse. [131] Placet igitur in his rebus aliquid imprudentia praeteriri ? Non placet, iudices, sed necesse est. Etenim si Iuppiter Optimus Maximus, cuius nutu et arbitrio caelum terra mariaque reguntur, saepe ventis vehementioribus aut immoderatis tempestatibus aut nimio calore aut intolerabili frigore hominibus nocuit, urbes delevit, fruges perdidit, quorum nihil pernicii causa divino consilio sed vi ipsa et magnitudine rerum factum putamus, at contra commoda quibus utimur lucemque qua fruimur spiritumque quem ducimus ab eo nobis dari atque impertiri videmus, quid miramur, iudices, L. Sullam, cum solus rem publicam regeret orbemque terrarum gubernaret imperique maiestatem quam armis 
receperat tum legibus confirmaret, aliqua animadvertere non potuisse? nisi hoc mirum est quod vis divina adsequi non possit, si id mens humana adepta non sit.

Si può capire che gli interpreti si siano posti il problema del tono di questo passo, che è apparso ambiguo e sarcastico ${ }^{5}$, giungendo a ipotizzare che sia stato aggiunto da Cicerone dopo il ritorno dalla Grecia e la morte di Silla ${ }^{6}$. Forse questo è eccessivo : la valutazione va fatta all'interno del flusso complessivo del discorso, considerando anche come un'anticipazione la si era avuta al capitolo 22 . Non c'è, pare, un'assimilazione alla divinità suprema, che è colta nelle sue valenze 'atmosferiche' ${ }^{17}$ è messo invece in evidenza il parallelismo nell'operare. L'alternanza delle condizioni atmosferiche che collaborano provvidenzialmente al bene dell'uomo, può essere sconvolta da alcuni eccessi, ma questi non compromettono la fede in una provvidenza che regge l'universo ${ }^{8}$.

Risulta del tutto evidente l'analogia dell'argomentazione con quella che Ovidio ci propone nel II libro dei Tristia. In entrambi i casi abbiamo il passaggio dalla concezione secondo cui Giove controlla il cosmo, ma non può essere ritenuto responsabile di ciò che di male tocca ad ogni singolo individuo, all'idea che il dittatore/l'imperatore non può sapere tutto quello che accade e intervenire di conseguenza.

osservato da tempo come Cicerone si collochi alle origini di molti temi e motivi della produzione panegiristica che conoscerà il suo sviluppo nell'età imperiale. In una certa misura questo si spiega bene con il fatto che l'oratore opera nel periodo delle grandi personalità che portano alla fine della repubblica. Gli elementi panegiristici in Cicerone sono stati di recente oggetto di attenzione ${ }^{9}$, ma potrebbero fornire spunto per ulteriori osservazioni. A ciò si aggiunge il rilievo delle orazioni cesariane per la definizione di una strategia nelle relazioni con il potere che sia utile anche per Ovidio nel suo esilio ${ }^{10}$.

Naturalmente l'onniscienza è legata alla tematica della giustizia fin da Omero ed Esiodo (Op. 238-243; 267-269) ${ }^{11}$, e la limitazione dell'intervento degli dèi a quanto vi è di significativo nell'esistenza del mondo è un pensiero che potremmo definire quasi proverbiale. È attestato nel dramma ${ }^{12}$, e in seguito è anche oggetto di riflessione filosofica : è un argomento discusso da Platone nelle Leggi (10,900c-903d $)^{13}$, ma con una soluzione diversa rispetto a quella adottata poi in àmbito peripatetico e soprattutto stoico. L'idea che la divina provvidenza si concentri sui grandi problemi piuttosto che su quelli dei singoli è originariamente stoica ${ }^{14}$, ma in età imperiale diviene un luogo comune (e. g. Iust. Trypho 1, 47). Già nella stessa Stoa, però, c'è un'oscillazione concettuale, che emerge in Cicerone nelle parole del portavoce di questa scuola nel libro II del De natura deorum : la provvidenza degli dèi veglia su ogni singolo individuo (164) ; gli dèi prendono in considerazione i grandi problemi e trascurano quelli piccoli (167). La discussione rimane aperta nel De providentia di Filone, un trattato centrale sull'argomento.

11 In particolar modo in àmbito peripatetico e stoico era stato inoltre riproposto il paragone con l'operare del magistrato o del sovrano : il sovrano o la legge non si curano dei casi singoli e dei piccoli dettagli ; per il parallelo a questo proposito tra dèi e sovrani terreni vd. [Aristot.] mund. 398a : il gran re forse si incarica dell'esecuzione dei suoi stessi ordini nelle sue vaste province ?; cfr. Cic. nat. deor. 3, 86; Sen. nat. 2, 46 ; Philo prov. 2, 99; 102 ; poi Max. Tyr. 5, $4^{15}$. Un concetto di questo tipo, che si oppone a un'immagine della provvidenza troppo asservita ai bisogni degli uomini, pare che tra i peripatetici fosse corrente : cfr. Alex. Aphr. fat. 30. Tuttavia utilizzare simili paragoni è 
nascondersi che ciò che è messo in conto alla maestà regale è condizionato in realtà dai limiti delle capacità umane, e che se il sovrano non si volge ad occuparsi delle cose minute è perché non ne ha la capacità. Ciò che non è possibile ai re umani dovrebbe esserlo al re celeste : Cic. nat. deor. 3, 90.

A una diversa linea concettuale fa riferimento l'idea espressa e sviluppata da Plutarco in connessione con Pericle, sia nella Vita di Pericle $(7,7)$, sia in praec. ger. reip. $15,811 \mathrm{~d}$ (dove peraltro è difficile stabilire se si tratti del pensiero di Pericle o di quello di Critolao $^{16}$ ) : ci si riferisce, anche con la citazione di Eur. fr. 974 Kannicht, al fatto che un uomo politico potente debba interessarsi soltanto dei problemi principali, e non di ogni cosa, al fine di non suscitare astio attraverso la sua onnipresenza.

L'importanza dello sviluppo di queste riflessioni è notevole, in quanto, come si è detto, il problema dell'onniscienza divina si collega strettamente a quello della giustizia: vedere, e quindi sapere ciò che accade, è un elemento da sempre in rilievo nelle discussioni sui maggiori meriti del sovrano, presente, rispetto agli dèi lontani: esemplare l'espressione del concetto nell'itifallico in onore di Demetrio Poliorcete (Hermocl. fr. 1, 15-18 Powell; vd. infra). La necessità di una vicinanza è messa efficacemente in evidenza in un frammento della Melanippe Desmotis di Euripide (506 Kannicht), dove si dice dell'opinione erronea secondo cui ci sarebbero delle tavolette su cui Zeus avrebbe scritto le colpe degli uomini ${ }^{17}$, mentre invece la Giustizia è vicina e tutto vede ${ }^{18}$.

Ovidio insiste pertanto su temi con una lunga storia e uno sviluppo consolidato ; erano però soggetti a vivo dibattito, ben connessi all'ideologia del servizio, che è quella che trovava espressione nei vari 'specchi dei principi' ellenistici. La problematicità della questione consente di inserire la riflessione all'interno dell'ortodossia ideologica imperiale senza esercitare un effetto corrosivo.

15 Per Cicerone l'impossibilità di occuparsi di ogni cosa è un elemento che entra all'interno di un discorso di difesa che non intende mettere in discussione l'ordinamento provvidenziale del cosmo. L'argomento giunge dal grande serbatoio dell'ideologia della monarchia per giustificare quella che si configura come una palese ingiustizia. Il peso della tradizione e l'esempio ciceroniano rinvigoriscono l'argomentazione di Ovidio, che vi aggiunge la forza del brano oraziano. È la statio del princeps, il suo compito provvidenziale, tema che è sviluppato con molte variazioni a tr. 2, 219-238, che impedisce ad Augusto di leggere l'Ars amatoria. Rimane notevole il fatto che alla fine del brano quanto emerge è il valore di exemplum morale che Augusto viene a rivestire, il modo in cui egli stesso deve essere condizionato dai suoi modelli di comportamento.

16 (II) Augusto, dunque, analogamente agli dèi, ignora elementi importanti per la valutazione della pena che ha inflitto al poeta. Può però essere al di sopra degli dèi nell'esercizio della giustizia.

Questo discorso conosce uno sviluppo in Pont. 3, 6.

Nella parte centrale dell'epistola (vv. 17-38) si mostra come anche coloro che sono colpiti dall'ira divina possano riprendersi, e gli dèi stessi non impediscano che siano soccorsi. Gli dèi non infieriscono senza fine sugli infelici, e tanto meno lo fa Augusto, che tempera la sua forza con la Giustizia, alla quale ha consacrato un tempio. Gli dèi colpiscono gli innocenti insieme ai colpevoli, per i quali, una volta morti, non c'è più 
alcun rimedio; Cesare invece ha reintegrato molti condannati togliendo loro parte della pena, e Ovidio prega di entrare in questo numero (vv. 27-38) :

Iuppiter in multos temeraria fulmina torquet, qui poenam culpa non meruere pati.

Obruerit cum tot saevis deus aequoris undis, ex illis mergi pars quota digna fuit?

Cum pereant acie fortissima quaeque, vel ipso iudice delectus Martis iniquus erit.

At si forte velis in nos inquirere, nemo est, qui se, quod patitur, commeruisse neget.

Adde quod extinctos vel aqua vel Marte vel igni nulla potest iterum restituisse dies :

restituit multos aut poenae parte levavit Caesar, et in multis me precor esse velit.

Come è noto, Pont. I-III presenta una forte compattezza strutturale, e la collocazione dell'elegia ne è una riprova, dato che vengono sviluppate importanti riflessioni di poetica, in particolare relativamente all'elemento che caratterizza la raccolta, la menzione esplicita del nome del singolo destinatario. Ovidio rovescia la prospettiva adottata finora : non dichiarare il nome del proprio destinatario è un atto di sfiducia nei confronti di Augusto, che, garante di moralità e cultore della giustizia, approva la fedeltà dimostrata all'esule dall'amico rimasto a Roma. Come in 1, 1 , si fa esplicito il confronto con il mondo divino: in quel caso (vv. 51-58) la clemenza del sovrano era paragonata a quella degli dèi, che graziano chi è stato da loro stessi punito; qui il parallelo dovrebbe tornare tutto a vantaggio e a gloria del princeps : Augusto è coinvolto indirettamente, ma in maniera decisa, nelle argomentazioni di Ovidio, in quanto deve esibire una razionalità e una clemenza che lo rendano superiore nella giustizia all'arbitrarietà dell'operare divino. È invece reso simile a un crudele tiranno proprio da coloro che hanno di lui timore. La disincantata amarezza che segna la parte finale di Pont. I-III si fa sentire però anche in questa epistola : porre se stesso come exemplum della clemenza di cui il princeps ha dato prova, in un simile contesto di messa in discussione delle azioni del mondo divino, non può non indurre il lettore alla riflessione. Tanto più forte, pertanto, è la pressione che viene esercitata su Augusto.

In questa elegia Ovidio dà l'impressione di voler giungere ai limiti di quanto gli è consentito dal sistema che egli stesso ha costruito. La negazione della caratteristica principale delle Ex Ponto, l'enunciazione del nome del destinatario, non può non produrre un risultato paradossale. L'aggressività che si rivolge contro l'amico pavido, che non vuole partecipare al progetto ovidiano, finisce per investire di riflesso anche il sovrano.

Vengono però messi in atto, in questo caso come in altri, una serie di elementi di compensazione ${ }^{19}$. Qui è rilevante il modo in cui è rappresentata la giustizia del princeps rispetto a quella degli dèi : Giove, Nettuno e Marte colpiscono alla cieca, laddove chi è stato punito da Augusto riconosce la propria colpa; inoltre la pena inflitta dall'imperatore può cessare, ma non così i colpi che si sono avuti dagli dèi (23-38).

Il ragionamento si colloca dunque in una dimensione diversa rispetto al precedente (ad Augusto sfuggono i dettagli come accade con gli dèi): la giustizia di Augusto $^{20}$ è superiore a quella divina, che evidentemente viene definita arbitraria. L'imperatore, del resto, è egli stesso sacrario della giustizia (vv. 25-26) ${ }^{21}$, un'immagine che può essere 
anche connessa alla problematica, in senso più stretto politico-religiosa, dell'edificazione di un tempio al sovrano ${ }^{22}$.

L'àmbito ideologico nel quale ci si muove recupera argomentazioni genericamente epicuree: la casualità con cui Giove scaglia i suoi fulmini ripropone un punto importante del De rerum natura: dopo aver spiegato l'origine di questi fenomeni celesti secondo la teoria atomica, Lucrezio contesta aspramente la concezione tradizionale che vedrebbe un'intenzionalità nel modo in cui i fulmini colpiscono gli uomini e le loro cose. Il primo argomento utilizzato è quello che ad essere colpiti sono individui del tutto innocenti, laddove questa sorte dovrebbe toccare ai malvagi $(6,387-395)$, un dato che era stato anticipato a 2,1103-1104 (saeviat exercens telum quod saepe nocentis / praeterit exanimatque indignos inque merentis) ${ }^{23}$. Si tratta, peraltro, di idee tradizionali, che possono essere fatte risalire fino a ciò che sostiene Socrate nelle Nuvole di Aristofane (398-402) e che in fondo danno voce ai ricorrenti dubbi sulla teodicea ${ }^{24}$. ̀̀ forse tuttavia significativo che influenze epicuree siano presenti nell'inno in onore di Demetrio Poliorcete (vv. 15-18) ${ }^{25}$, che rappresenta un episodio così emblematico nello sviluppo della concezione della divinità del sovrano. In quel caso il dato decisivo è la 'presenza' : il monarca è vicino e si cura dei suoi sudditi, laddove gli dèi sono lontani. Questo elemento, che è in genere quello su cui si fonda la superiorità del re terreno, conosce un'espressione notevole in Ov. tr. 4, 4, 20 quorum hic aspicitur, creditur ille deus. Il motivo viene rovesciato nella prospettiva dell'esilio : dalla lontana Tomi anche Augusto è un absens numen, che però proprio in questo modo può essere del tutto identificato negli dèi : tr. 5, 2, 45-54

Alloquor en absens absentia numina supplex, si fas est homini cum Iove posse loqui.

Arbiter inperii, quo certum est sospite cunctos Ausoniae curam gentis habere deos,

o decus, o patriae per te florentis imago, o vir non ipso, quem regis, orbe minor

(sic habites terras et te desideret aether, sic ad pacta tibi sidera tardus eas)

parce, precor, minimamque tuo de fulmine partem

deme : satis poenae, quod superabit, erit ${ }^{26}$.

L'elaborazione dei motivi e delle argomentazioni ovidiane è connessa con la necessità apologetica e con l'obiettivo concreto che l'esule si propone. Funzionale a questo è la definizione della figura dell'imperatore come supremo e unico giudice, che viene dunque sottratto all'àmbito della casualità e rappresenta l'istanza definitiva della giustizia. Ovidio in realtà non ci dice se Augusto sia davvero tale o no. Questo è il modello che viene imposto al sovrano, al quale egli deve adeguarsi, in base ad un implicito ricatto, e questa è la funzione che ha il recupero della tradizione relativa alla superiore capacità di intervento di chi governa sulla terra. Cosa succederebbe se Augusto non corrispondesse alle attese di una giustizia più vera? Diventerebbe come gli dèi inaffidabili e arbitrari che dominano la scena delle altre opere ovidiane, prime fra tutte le Metamorfosi. I modelli di comportamento che sono proposti sono quelli della più elevata possibilità di celebrazione per l'imperatore e sono portatori di un'istanza persuasiva (in questo senso hanno una valenza 'retorica') : mostrano al potere come dovrebbe configurarsi per essere all'altezza delle attese dei sudditi, per essere davvero considerato legittimo. Se pensiamo all'attenzione con cui il princeps ha costruito la sua figura, il tentativo di Ovidio poteva non essere considerato come volto in partenza al fallimento ${ }^{27}$. 


\section{BIBLIOGRAPHIE}

D. Babut, Plutarque et le Stoïcisme, Paris 1969.

A. Barchiesi, Insegnare ad Augusto : Orazio, Epistole 2, 1 e Ovidio, Tristia II, « Mater. e Discuss. » 31, 1993, pp. 149-184.

A. Barigazzi, La formazione spirituale di Menandro, Torino 1965.

G. Bauer, Claustrum animae, München 1973.

J. Benedum, Studien zur Dichtkunst des späten Ovid, Diss. Gießen 1967.

D. H. Berry, The Publication of Cicero's Pro Roscio Amerino, « Mnem. » 57, 2004, pp. 80-87.

F. Bessone, La Tebaide di Stazio. Epica e potere, Pisa-Roma 2011.

I. Ciccarelli, Citra necem tua constitit ira : le ambigue manifestazioni della clementia di Augusto verso Ovidio, « Aufidus » 43, 2002, pp. 23-32.

I. Ciccarelli, Commento al II libro dei Tristia di Ovidio, Bari 2003.

A. Dieterich, Nekyia, Leipzig-Berlin $1913^{2}$.

A. R. Dyck, Cicero, Pro Sexto Roscio, Cambridge 2010.

H. Flasche, Similitudo templi (Zur Geschichte einer Metapher), « Deutsche Vierteljahrsschr. Literaturwiss. Geistesgesch. » 23, 1949, pp. 81-125.

G. Focardi, Difesa, preghiera, ironia nel II libro dei Tristia di Ovidio, « Stud. Ital. Filol. Class. » 47, 1975, pp. 86-129.

A. F. Garvie, Aeschylus, Coephori, edited with introduction and commentary, Oxford 1986.

F. Gasti, Introduzione a Cicerone, Orazioni cesariane, Milano 1997.

Ch. Gnilka, Studien zur Psychomachie des Prudentius, Wiesbaden 1963.

A. W. Gomme-F. H. Sandbach, Menander. A Commentary, Oxford 1973.

I. Gradel, Emperor Worship and Roman Religion, Oxford 2002.

H. Hanse, Gotthaben in der Antike und im frühen Christentum, « Relig. Vortr. Vorarb. » 27, Berlin 1939.

P. Hardie, Ovid's Poetics of Illusion, Cambridge 2002.

J. Ingleheart, A Commentary on Ovid, Tristia, Book 2, Oxford 2010.

D. Kienast, Augustus, Darmstadt 1982.

A. J. Kronenberg, Ad Plutarchi Moralia, « Mnem. » 52, 1924, pp. 61-112.

M. Labate, Elegia triste ed elegia lieta. Un caso di riconversione letteraria, « Mater. e Discuss. » 19, 1988, pp. 91-129.

K. Latte, Römische Religionsgeschichte, München 1960.

D. S. Levene, God and Man in the Classical Latin Panegyric, « Proc. Cambr.Philol. Soc. » 43, 1997, pp. 66-103.

M. Marcovich, Hermocles' Ithyphallus for Demetrius, in Id., Studies in Graeco-Roman Religions and Gnosticism, Leiden-New York-København-Köln 1988, pp. 8-19. 
R. H. Martin-A. J. Woodman, Tacitus, Annals, Book IV, Cambridge 1989.

M. M. McGowan, Ovid in Exile, Leiden-Boston 2009.

S. Morton Braund, Praise and Protreptic in Early Imperial Panegyric : Cicero, Seneca, Pliny, in M. Whitby (ed.), The Propaganda of Power. The Role of Panegyric in Late Antiquity, Leiden-Boston-Köln 1998, pp. 53-76.

E. Narducci, Cicerone. La parola e la politica, Roma-Bari 2009.

A. S. Pease, M. Tulli Ciceronis De natura deorum, I-II, Cambridge Mass. 1958.

S. Rochlitz, Das Bild Caesars in Ciceros "OrationesCaesarianae». Untersuchungen zur »clementia" und "sapientia Caesaris«, Frankfurt am Main-Berlin-Bern-New York-Paris-Wien 1992.

A. Traina, Lo stile "drammatico" del filosofo Seneca, Bologna $1995^{4}$.

H. S. Versnel, Religious mentality in ancient prayer, in Id. (ed.), Faith, hope and worship. Aspects of religious mentality in the ancient world, Leiden 1981, pp. 1-64.

J. H.Waszink, Q. Septimi Florentis Tertulliani, De anima, edited with introduction and commentary, Amsterdam 1947.

F. Wehrli, Die Schule des Aristoteles. Texte und Kommentar, X, Basel-Stuttgart $1969^{2}$.

O. Weinreich, Stiftung und Kultsatzungen eines Privatheiligtums in Philadelpheia in Lydien, « Sitz. Ber. Heidelberg » 1919, 16.

S. Weinstock, Divus Julius, Oxford 1971.

G. Wissowa, Religion und Kultus der Römer, München $1912^{2}$.

\section{NOTES}

1. M. Labate, Elegia triste ed elegia lieta. Un caso di riconversione letteraria, « Mater. e Discuss. »19, 1988, p. 120.

2. Sul passo e il confronto con Orazio cfr. A. Barchiesi, Insegnare ad Augusto: Orazio, Epistole 2, 1 e Ovidio, Tristia II, « Mater. e Discuss. » 31, 1993, pp. 159-165. Notevole il senso di orgoglio e cautela che traspare da epist. 1, 13, 17-18 carmina quae possint oculos auresque morari / Caesaris, sempre nel contesto delle istruzioni sul modo in cui avvicinare Cesare.

3. Il modo di rapportarsi a quello che è l'ordine di priorità valido per un potente è centrale nell'epistola alla moglie all'inizio del III libro delle Ex Ponto in riferimento a Livia (vv. 129-144).

4. Presente anche in Pont. 1, 7, 43 qui pervidet omnia Caesar, dove il riferimento è alla capacità che Augusto possiede di giudicare correttamente a proposito della colpa di Ovidio.

5. Cfr. la nota ad loc. del commento di A. R. Dyck, Cambridge 2010, pp. 186-187.

6. D. H. Berry, The Publication of Cicero's Pro Roscio Amerino, « Mnem.» 57, 2004, pp. 80-87. L'ipotesi della rielaborazione in un secondo tempo con l'aggiunta dei passi più audaci è definita attraente, ancorché impossibile da verificare, da E. Narducci, Cicerone. La parola e la politica, RomaBari 2009, pp. 47-48.

7. Così in Pont. 3, 6, 27-32 (vd. infra) i nomi degli dèi hanno valore di metonimia.

8. Forse è presente anche l'idea che la divinità possa conferire alcuni compiti a demoni malvagi, e questo sarebbe come se un re affidasse incarichi amministrativi a generali e governatori perfidi e stupidi, che si divertissero a tormentare le persone per bene, secondo quanto osserva Plutarco nella sua polemica contro lo stoico Crisippo (stoic. repugn. 37, 1051c, SVF II 1178) ; cfr. anche D. 
Babut, Plutarque et le Stoïcisme, Paris 1969, p.291, con ulteriori indicazioni sui demoni come satrapi.

9. D. S. Levene, God and Man in the Classical Latin Panegyric, « Proc. Cambr. Philol. Soc. » 43, 1997, pp. 66-103 (soprattutto sulla Pro Marcello); S. Morton Braund, Praise and Protreptic in Early Imperial Panegyric: Cicero, Seneca, Pliny, in M. Whitby (ed.), The Propaganda of Power. The Role of Panegyric in Late Antiquity, Leiden-Boston-Köln 1998, pp.53-76, in particolare sulla De imperio Cn. Pompei; F. Gasti, Introduzione a Cicerone, Orazioni cesariane, Milano 1997, pp. 21-24. In generale, e non solo per le cesariane, cfr. S. Rochlitz, Das Bild Caesars in Ciceros "Orationes Caesarianae» . Untersuchungen zur »clementia» und »sapientia Caesaris», Frankfurt am Main-Berlin-Bern-New York-Paris-Wien 1992, che considera questo gruppo di orazioni come un'articolazione del tema dello speculum principis.

10. Il rapporto con la Pro Ligario è messo in particolare evidenza in J. Ingleheart, A Commentary on Ovid, Tristia, Book 2, Oxford 2010, pp. 13-15. Materiale utile in G. Focardi, Difesa, preghiera, ironia nel II libro dei Tristia di Ovidio, in « Stud. Ital. Filol. Class. » 47, 1975, pp. 86-129.

11. Cfr. anche Aesch. Coeph. 61-65, però con problemi di testo : vd. la nota di A. F. Garvie, Oxford

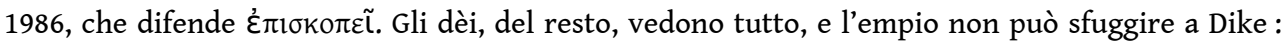
Plat. leg. 10, 901d ; 905a ; Xen. an. 2, 5, 7 ; Plut. superst. 4, 166d.

12. Eur. fr. 974 Kannicht; tr. anon. 448 Kannicht-Snell ; Men. Epitr. 1084-1091: il passo di Menandro, di cui possediamo il contesto, ci dà un'idea di come simili affermazioni conoscessero un uso argomentativo, senza inserirsi in un'ideologia coerente. Ci sono stati vari tentativi di rinvenirvi l'espressione di una filosofia: A. Barigazzi, La formazione spirituale di Menandro, Torino 1965, pp. 198-212, a sua volta ipotizza un'ascendenza aristotelica, ma vd. il commento ad loc. di A. W. Gomme-F. H. Sandbach, Oxford 1973, pp. 377-378.

13. Cfr. anche resp. 4, 420b-421e, e le successive trattazioni di ambito platonico : Philo prov. 2, 99 ; [Plut.] fat. 9-11, 572f-574e ; Apul. Plat. 1, 12.

14. Cic. nat. deor. 2, 167, con l'ampia nota di A. S. Pease, Cambridge Mass. 1958 ; 3, 86 ; Arr. Epict. 1, 12, 2. Cfr. anche e. g. SVF II $1169 ; 1171 ; 1174 ; 1176 ; 1181 ; 1184$; M. Aur. 5, 8, e Cic. nat. deor. 2, 73-75. La divina provvidenza e la sua preoccupazione per l'interesse della collettività rispetto a quello dei singoli, è oggetto di una riflessione anche in Max. Tyr. 41, 4.

15. Vd. anche Philo prov. 2, 15 ; [Plut.] fat. 4, 569d-570a ; Alcin. 26.

16. Per F. Wehrli è il fr. 37 di Critolao (Die Schule des Aristoteles. Texte und Kommentar, X, Basel-

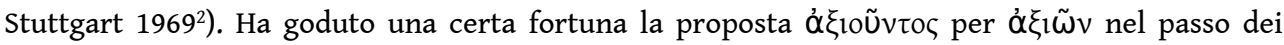
Moralia, che attribuirebbe il pensiero a Pericle e non più a Critolao, avanzata da A. J. Kronenberg, «Mnem. » 52, 1924, pp. 91-92. Per molti aspetti un'inversione di modelli, per cui quello umano è proiettato su quello divino, è nella rappresentazione paradossale di Zeus travolto dal cumulo dei suoi impegni, non solo generali ma anche minuti, all'inizio del Bis accusatus di Luciano.

17. Per l'immagine, anche proverbiale, del libro nel quale sono scritte le azioni degli uomini cfr. A. Dieterich, Nekyia, Leipzig-Berlin $1913^{2}$, p. 127 ; per Eschilo cfr. fr. 281a, 21 R.

18. Utili indicazioni in H. S. Versnel, Religious mentality in ancient prayer, in Id. (ed.), Faith, hope and worship. Aspects of religious mentality in the ancient world, Leiden 1981, pp. 28-31; buona raccolta di passi in O. Weinreich, Stiftung und Kultsatzungen eines Privatheiligtums in Philadelpheia in Lydien, «Sitz. Ber. Heidelberg » 1919, 16, pp. 61-62.

19. Un esempio : un concetto di cui Ovidio si serve per spiegare il comportamento di Augusto nei suoi confronti, espressione di un difficilissimo equilibrio, è la riluttanza a punire che sarebbe dimostrata dall'imperatore, analoga a quella di Giove che spesso tuona senza colpire con il fulmine (e. g. tr. 2, 33-36 ; Pont. 2, 2, 116) : sul passo dei Tristia, ma con osservazioni valide a livello generale, cfr. I. Ciccarelli, Citra necem tua constitit ira : le ambigue manifestazioni della clementia di Augusto verso Ovidio, in "Aufidus» 43, 2002, pp. 23-32; vd. anche il commento a Tristia II, Bari 2003, ad loc. Naturalmente, c'è un'evidente contraddizione, a prima vista irriducibile, tra tanta professione di clemenza e l'ira che si è manifestata nel colpire l'error del poeta, una 
contraddizione che sta alla base di tutta la problematica dell'esilio. Ovidio è stato punito - è un dato di fatto - da un numen iratum, e questa potrebbe sembrare una caratterizzazione univocamente negativa del suo giudice. Ma il dio si è adirato con lui giustamente (merito), anzi l'ira è stata inferiore a quanto ci si potesse attendere. Augusto è stato costretto ad intervenire contro Ovidio.

20. Ovidio sottolinea più volte questa particolare virtù dell'imperatore (la terza sul clupeum virtutis) : Pont. 2, 1, 33-34; tr. 4, 4, 12 ; met. 15, 833. Sulla Iustitia Augusti da un punto di vista cultuale cfr. G. Wissowa, Religion und Kultus der Römer, München $1912^{2}$, p. 332 ; K. Latte, Römische Religionsgeschichte, München 1960, p. 300 e RE X. 2, 1339 con bibl.; S. Weinstock, Divus Julius, Oxford 1971, p. 247 ; D. Kienast, Augustus, Darmstadt 1982, p. 83. Una rassegna delle varie opinioni su ciò che sia stato effettivamente innalzato alla Iustitia, se un tempio, un altare o una statua, in J. Benedum, Studien zur Dichtkunst des späten Ovid, Diss. Gießen 1967, pp. 90-95: la conclusione è che si tratterebbe di una statua che sanciva il completamento o la consacrazione di un tempio realizzato prima dell's gennaio del 13 d. C. Sull'epiteto iustus riferito ad Augusto in Ovidio, cfr. M. M. McGowan, Ovid in Exile, Leiden-Boston 2009, p. 141.

21. La giustizia ha un tempio nella mente di Augusto : Pont. 2, 1, 33-34 Iustitiam ... sui caste placasse parentis, / illo quae templum pectore semper habet. In questa immagine confluiscono vari filoni concettuali : in primo luogo quello relativo al deus internus (cfr. J. Haußleiter, RLAC III, 794-842), presente, in particolare, in Posidonio (cfr. anche Seneca fr. 88 Vottero), ma in sostanza già nella problematica platonica sull'anima. In Filone, poi, il motivo conoscerà un certo sviluppo, anche con metafore edilizie, in relazione al voũ $\varsigma$ che abita nell'anima. A ciò vanno aggiunte la discussione medica sulla sede dell'anima (Tert. anim. 15, 5 con la nota ad loc. di J. H. Waszink, Amsterdam 1947) e le credenze popolari (papiri magici), poi filtrate dalla filosofia, e. g. la Stoa più recente : presenze divine personali (e. g. Eros o Hermes) erano concepite come presenti nel cuore

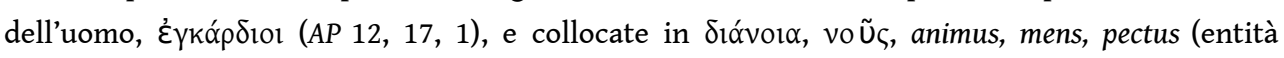
strettamente associate per via di metonimia) ; "avere in sé la divinità" è analizzato in senso più strettamente religioso in H. Hanse, Gott haben in der Antike und im frühen Christentum, «Relig. Vortr. Vorarb. » 27, Berlin 1939. Così è posta la base per la metafora del tempio : Lucr. 5, 102-103 via qua munita fidei / proxima fert humanum in pectus templaque mentis; Val. Max. 4, 7 ext. 1 ; Tac. ann. 4, 38, 1 [i benefici di Tiberio verso gli uomini] haec mihi in animis vestris templa, hae pulcherrimae effigies et mansurae (con la nota di R. H. Martin-A. J. Woodman, Cambridge 1989) ; cfr. ancora G. Bauer, Claustrum animae, München 1973; A. Traina, Lo stile "drammatico" del filosofo Seneca, Bologna $1995^{4}$, p. 76 ; una storia della metafora dall'antichità ai tempi moderni in H. Flasche, Similitudo templi (Zur Geschichte einer Metapher), «Deutsche Vierteljahrsschr. Literaturwiss. Geistesgesch. » 23, 1949, 81-125. L'influenza del pensiero stoico nei passi ovidiani è maggiormente sottolineata da Ch. Gnilka, Studien zur Psychomachie des Prudentius, Wiesbaden 1963, pp. 83-84 ; cfr. anche O. Michel, ThW IV, 890-891.

22. Cfr. I. Gradel, Emperor Worship and Roman Religion, Oxford 2002, pp. 73-77.

23. È interessante che il primo esempio sia propriamente quello di Iuppiter Tonans, a proposito del quale vanno ricordati l'aneddoto relativo ad Augusto, che stava appunto per essere colpito da un fulmine durante la guerra contro i Cantabri, e il culto da lui incrementato. Sul tempio cfr. P. Gros, in E. M. Steinby (ed.), Lexicon Topographicum Urbis Romae, III, Roma 1996, pp. 159-160.

24. Sulla casualità nella caduta dei fulmini cfr. anche Cic. div. 2, 44-45. Vd.inoltre Lucian. Iupp. conf. 16 ; Lucan. 7, 448 : Giove non interviene benché abbia i suoi fulmini.

25. Cfr. M. Marcovich, Hermocles' Ithyphallus for Demetrius, in Id., Studies in Graeco-Roman Religions and Gnosticism, Leiden-New York-København-Köln 1988, p. 14.

26. Sviluppi particolarmente concettosi del motivo in Pont. 2, 8 : cfr. P. Hardie, Ovid's Poetics of Illusion, Cambridge 2002, pp. 318-322. Per la poesia di età imperiale utile F. Bessone, $L a$ Tebaide di Stazio. Epica e potere, Pisa-Roma 2011, pp. 45-74, in particolare 65-66. 
27. Ringrazio gli amici Sergio Casali e Andrea Cucchiarelli, e gli anonimi referee della rivista per la loro attenta lettura.

\section{RÉSUMÉS}

Nella poesia dell'esilio Ovidio sviluppa una linea di difesa proponendo e imponendo ad Augusto quanto è proclamato dalla stessa ideologia imperiale, i cui elementi vengono scomposti e assemblati in base alle necessità dell'argomentazione apologetica. La mancata conoscenza dell' Ars amatoria da parte di chi lo ha condannato, è giustificata con il confronto con Giove, secondo uno sviluppo già ciceroniano ; altrove invece si celebra la giustizia del princeps come superiore a quella divina. I modelli di comportamento proposti sono quelli della più elevata possibilità di celebrazione per l'imperatore e sono portatori di un'istanza persuasiva (in questo senso hanno una valenza 'retorica'): mostrano al potere come dovrebbe configurarsi per essere davvero considerato legittimo.

\section{INDEX}

Mots-clés : Ovidio, esilio, Epistulae ex Ponto, Augusto, iustitia.

\section{AUTEUR}

\section{LUIGI GALASSO}

Università di Pavia 